\title{
Supervised Physical Therapy Versus Video- Assisted Technique for Chronic Mechanical Low Back Pain: A Comparative Study
}

\author{
Spoorthi Shetty ${ }^{1}$ Dhanesh Kumar KU ${ }^{1}$ Purusotham Chippala ${ }^{1}$ \\ ${ }^{1}$ Nitte Institute of Physiotherapy, Deralakatte, Mangalore, Karnataka, \\ Address for correspondence Dhanesh Kumar KU, MPT, PhD, Nitte \\ India \\ Institute of Physiotherapy, Deralakatte, Mangalore 575018 , \\ Karnataka, India (e-mail: spoorthishetty252@gmail.com).
}

J Health Allied Sci ${ }^{\mathrm{NU}}$ 2022;12:277-279.

\begin{abstract}
Objective To compare the effect of supervised physical therapy versus video-assisted technique in patients with chronic mechanical low back pain.

Materials and Methods This is a comparative study. Forty-two patients were recruited in the study where they were randomized by simple random sampling. Group A $(n=21)$ received supervised physical therapy, and Group B $(n=21)$ received the video-assisted technique. The intervention was given 45 minutes per day for 15 days. The measures of visual analog scale (VAS) and Roland-Morris Disability

Keywords

- mechanical low back pain

- supervised physical therapy

- home exercise

- video-assisted technique Questionnaire (RMQ) were taken both at baseline and after 15 days of intervention.

Results Comparison between the two groups using Mann-Whitney U test, supervised physiotherapy group showed significant improvement in VAS on activity, RMQ, and RMQ percentage $(p<0.005)$. However, Group A, VAS on rest was not significant $(p>0.005)$.

Conclusion Supervised physical therapy is effective in reducing pain on activity and improved the disability of patients with chronic mechanical low back pain than the video-assisted technique.
\end{abstract}

\section{Introduction}

Mechanical back pain is the most prevalent type of back pain in musculoskeletal disorders. ${ }^{1}$ According to epidemiological research, over $95 \%$ of the world's population suffers from mechanical low back discomfort. ${ }^{2}$ Mechanical low back pain (MLBP) costs industrialized countries a lot of money on a global scale. It has one of the most expensive treatments, resulting in considerable direct medical expenses as well as indirect social costs such as lost productivity, disability, and workers' compensation claims. ${ }^{3,4}$ MLBP is recognized by the presence or absence of signs and symptoms with workrelated issues, different postural defects, and its move- ments. ${ }^{5,6}$ Supervised physical therapy is where the physical therapist is physically present on the premises and immediately available for direction and supervision. Supervised physical therapy needs certain available resources, time, and cost, which may sometimes not be affordable with the patients or applied in clinical settings. ${ }^{7}$

Unsupervised training or video-assisted training comes as an attractive, low-cost alternative for supervised physical therapy that a patient can perform by looking at videos that consist of exercises related to the condition. ${ }^{8}$

Studies have shown that supervised physical therapy is better than unsupervised physical therapy. ${ }^{9}$ Patients suffering from low back pain as a result of the COVID-19 pandemic published online December 30, 2021
DOI https://doi.org/

$10.1055 / \mathrm{s}-0041-1740330$. ISSN 2582-4287.

\footnotetext{
(C) 2021. Nitte (Deemed to be University). All rights reserved. This is an open access article published by Thieme under the terms of the Creative Commons Attribution-NonDerivative-NonCommercial-License, permitting copying and reproduction so long as the original work is given appropriate credit. Contents may not be used for commercial purposes, or adapted, remixed, transformed or built upon. (https://creativecommons.org/ licenses/by-nc-nd/4.0/)

Thieme Medical and Scientific Publishers Pvt. Ltd., A-12, 2nd Floor, Sector 2, Noida-201301 UP, India
} 
or due to other reasons are unable to attend hospital therapy, demanding the use of a video-assisted technique. As a result, the study's goal is to determine if video-assisted techniques are beneficial when compared with supervised techniques.

\section{Materials and Methods}

\section{Trial Setting}

A comparative study was conducted from April 2020 to April 2021 at Justice K. S. Hedge Charitable Hospital, Mangalore, Karnataka. The ethical clearance for the proposed study was acquired from Nitte Institute of Physiotherapy's Institutional Ethics Committee, Mangalore, Karnataka, India on March 3, 2020, with reference no NIPT/IEC/Min/20/20192020. The study was registered under the CTRI number CTRI/ 2020/11/029126. This trial followed the ethics of research in humans (Helsinki convention norms) and the participants were informed about the process of study and consent was obtained. The participants were also made known about their rights to withdraw anytime their participation from the study. Materials that were used for research included the screening tool, data collection sheets, informed consent forms, pocket dairy, paper, pen, smartphones, and mat.

The participants were randomly allocated to two groups. Group A received thermotherapy, hamstring, and back muscles stretching and core stability exercises were given as a part of the conventional treatment under the supervision of a physical therapist, whereas Group B received the same as above but in the form of a video. VAS was used for pain and disability was measured by RMQ.

\section{Group A}

The treatment was delivered to the subjects under the supervision of a physical therapist. Outcome measures VAS and RMQ were taken before starting the treatment and after 2 weeks.

Patient education and ergonomic advices were given in the beginning of study. Moist heat application was given for 10 minutes, Hamstring and back muscles stretching-patient was asked to sit in a long sitting position with knees fully extended and feet together free or plantar flexion. Then, the patient was asked to bend forward from the hip to reach toward the feet with the head in flexion, hold for 30 seconds, repeat for 3 sets of 10 repetitions, and rest time for 30 seconds.

Core stability exercises: Abdominal tuck-in: The patient was asked to be in a crook lying position with the arms beside the body. Pull the umbilicus toward the spine and hold for 10 seconds (3 sets), 10 repetitions. Straight leg raise: The patient should be lying in the supine position with arms beside the body and was asked to lift one leg for $\sim 15 \mathrm{~cm}$ off the floor and hold for 10 seconds and return to the position. Repeat the exercise on the other side as well. Three sets (10 repetitions). Bridging: The patient was asked to lie in the supine crook position with arms beside and was asked to raise the hip and contract the abdomen, keeping feet flat on the floor and hold for 10 seconds and return to the same position and relax the abdomen (3 sets, 10 repetitions). Plank: The patient was asked to get in a push-up position and with forearms on the ground instead of hands, the patient was asked to tighten the abdominals keeping the neck and spine in a neutral position by creating a straight line from the head to toe, hold for 5 to 10 seconds, 10 repetitions, 3 sets. Bird dog exercise: The patient was asked to get into the quadruped position and asked to pull the abdomen into the spine, keeping the back and pelvis still and stable. The patient was asked to raise one arm and opposite leg and hold for 5 to 10 seconds and return to the starting position and repeat with the other side (10 repetitions, 3 sets).

\section{Group B}

Patients received the same exercises as Group A but in the video form; VAS and RMQ were taken before the study and after 2 weeks. Here, the patient was called for follow-up and again all outcome measures were taken to evaluate the results. Patients were given a pocket diary and a pen to note down the date and time whenever they performed exercise.

\section{Results}

Data analysis was performed by SPSS (version 17) for Windows. Alpha value was set as 0.05 . Descriptive statistics were performed to calculate the mean and standard deviation for demographic variables and outcome variables. Unpaired $t$-test was used to compare demographic variables, such as age. A Chi-square test was used to compare the gender distribution between the groups. Mann-Whitney $U$ test was used to identify differences in scores between the groups for VAS on rest, VAS on activity, RMQ and RMQ percentage. Wilcoxon signed rank-sum test was used to identify significant differences within groups for VAS on rest, VAS on activity, and RMQ. Microsoft Excel and Word were used to generate graphs and tables. Demographic baseline data are mean \pm standard deviation (SD). In Group A, the mean and SD of age was $37 \pm 12.16$ and in Group B, the mean and SD of age was $28.86 \pm 9.68$. In Group A, there were 10 males and 11 females and in Group B, there were 6 males and 15 females. Both age and gender were homogenous among both groups. When compared between groups, the mean VAS on rest post score in Group A was 3.00 with a standard deviation of 1.09 and the mean VAS on rest post score in Group B was 3.05 with a standard deviation of 1.20 , which was not statistically significant ( $p>0.861$; MannWhitney $U$ value was -0.61632 ). The mean VAS on activity post score in Group A was 4.00 with a standard deviation of 1.304 and the mean VAS on activity post score in Group B was 4.90 with a standard deviation of 0.889 , which was statistically significant $(p<0.022$; Mann-Whitney $U$ value was -2.12566$)$. The mean RMQ post score in Group A was 5.62 with a standard deviation of 1.80 and the mean RMQ post score in Group B was 7.43 with a standard deviation of 1.75 , which was statistically significant ( $p<0.003$; Mann-Whitney $U$ value was -2.89291$)$ within the group was analyzed using the Wilcoxon test.

\section{Discussion}

The current study was designed to evaluate the effect of supervised physical therapy over video-assisted technique on patients with chronic MLBP. 
The researcher took 1 year to complete the study. In total, 108 subjects were screened for the study; 47 participants had difficulty in traveling to and from the hospital, 28 participants did not respond, 7 participants did not use smartphones, and 26 participants had other reasons. The screening tool included the demographic data, history of back pain, pain assessment VAS and RMQ for both pain and disability. In total, 42 patients were included in the study, 21 in each group, Group A (supervised physical therapy) and Group B (video-assisted technique) for 15 days. The outcome measures VAS and RMQ were taken on the first day and 15th day. A comparison between the groups showed that VAS on activity, RMQ, and RMQ percentage were having significant $(p<0.05)$, whereas VAS on rest was not having significant differences $(p>0.05)$.

The present study revealed a significant decrease in the RMQ percentage and RMQ score in Group A (supervised physical therapy) when compared with Group B (videoassisted technique). The results have been supported by Matarán-Peñarrocha et $\mathrm{al}^{9}$ and Will et $\mathrm{al}^{10}{ }^{10}$ on supervised physical therapy and unsupervised physical therapy on chronic MLBP where patients with persistent low back pain who got supervised exercise improved more in all patient-rated outcomes in short- and long-term than those who did not.

This study showed that supervised physical therapy resulted in better improvement than video-assisted technique $(p<0.05)$. The same was reported by Shahzad et al ${ }^{11}$ and Coulombe et al ${ }^{12}$ whose study was conducted on weight lifters injuries by dividing them into two groups where one was under the supervision and the other was one under nonsupervised; the results showed a significant improvement in supervised physical therapy by evaluating the Nordic musculoskeletal questionnaire, which revealed less injury under supervision. Hence, the present study showed significant result as in the Coulombe et al study. ${ }^{12}$

Unsupervised or video-assisted physical therapy is less beneficial than supervised physical therapy because the physical therapist will supervise and motivate the patient to perform the exercise with appropriate guidance and help the patient to perform the exercise on a regular basis, whereas in unsupervised or video-assisted physical therapy, the patient may lack motivation and may not continue the exercise.

\section{Conclusion}

The present study demonstrates that both supervised physical therapy and a video-assisted technique help alleviate pain and disability in chronic MLBP. However, comparing both groups, supervised physical therapy has better improvement than video-assisted technique in chronic MLBP. In supervised physical therapy, the physical therapist will supervise and motivate the patient to perform the exercise with appropriate guidance and help the patient to perform the exercise on a regular basis. However, due to personal circumstances, such as a pandemic, due to which a few patients complained about not being able to make it to the hospital on time and other personal reasons; so at this time, the visualassisted technique could be useful.

\section{Conflict of Interest}

None declared.

\section{References}

1 Borenstein D. Mechanical low back pain-a rheumatologist's view. Nat Rev Rheumatol 2013;9(11):643-653

2 Emami F, Yoosefinejad AK, Razeghi M. Correlations between core muscle geometry, pain intensity, functional disability and postural balance in patients with nonspecific mechanical low back pain. Med Eng Phys 2018;60:39-46

3 Haines T, Bowles KA. Cost-effectiveness of using a motion-sensor biofeedback treatment approach for the management of subacute or chronic low back pain: economic evaluation alongside a randomised trial. BMC Musculoskelet Disord 2017;18(01):18

4 Garcia AN, Costa LDCM, Hancock MJ, et al. McKenzie method of mechanical diagnosis and therapy was slightly more effective than placebo for pain, but not for disability, in patients with chronic non-specific low back pain: a randomised placebo controlled trial with short and longer term follow-up. Br J Sports Med 2018;52(09):594-600

5 Endean A, Palmer KT, Coggon D. Potential of magnetic resonance imaging findings to refine case definition for mechanical low back pain in epidemiological studies: a systematic review. Spine 2011; 36(02):160-169

6 Kumar SP. Efficacy of segmental stabilization exercise for lumbar segmental instability in patients with mechanical low back pain: a randomized placebo controlled crossover study. N Am J Med Sci 2011;3(10):456-461

7 Minetama M, Kawakami M, Teraguchi M, et al. Supervised physical therapy vs. home exercise for patients with lumbar spinal stenosis: a randomized controlled trial. Spine J 2019;19(08): 1310-1318

8 Piccoli L. Supervised and unsupervised physical exercise program in the treatment of chronic low back pain: randomized clinical trial. Journal of Engineering Research and Application. 2019;19 (02):16-23

9 Matarán-Peñarrocha GA, Lara Palomo IC, Antequera Soler E, et al. Comparison of efficacy of a supervised versus non-supervised physical therapy exercise program on the pain, functionality and quality of life of patients with non-specific chronic low-back pain: a randomized controlled trial. Clin Rehabil 2020;34(07): 948-959

10 Will JS, Bury DC, Miller JA. Mechanical low back pain. Am Fam Physician 2018;98(07):421-428

11 Shahzad H, Jabeen H, Arslan HR, et al. Musculoskeletal injuries among weight lifters with or without supervision? a comparative cross sectional study. The International Journal of Frontier Sciences 2021;5(02). Doi: 10.37978/tijfs.v5i2.368

12 Coulombe BJ, Games KE, Neil ER, Eberman LE. Core stability exercise versus general exercise for chronic low back pain. J Athl Train 2017;52(01):71-72 